\title{
Two consecutive myocardial tissue insults for inpatients with COVID-19
}

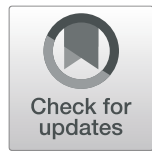

\author{
Minghua Su ${ }^{1 \dagger}$, Jieru Peng ${ }^{2 \dagger}$, Mengjun $\mathrm{Wu}^{3^{*}}$, Wuquan Deng ${ }^{4^{*}}$, Yousheng Yang $^{5^{*}}$ and Yong G. Peng ${ }^{6^{*}}$
}

The new coronavirus (COVID-19) originated in Wuhan, China, and has expeditiously spread across the global [1, 2]. Recent reports have suggested that myocardial injury was significantly associated with the fatality of the COVID-19 infection [3]. However, details of the risk factors leading to myocardial injury and death in adult inpatients with COVID-19 have not yet been well-described.

This was a retrospective case-control study $(n=105)$ with a confirmed novel coronavirus-infected pneumonia diagnosis between January 10, 2020, and February 20, 2020. It was approved by the institutional ethics board of The Fifth Hospital of Wuhan, Wuhan, P.R. China. Each individual non-survivor $(n=35)$ was matched with two subjects $(n=70)$ based on age, sex, and date of admission among discharged inpatients.

The non-survivors $(n=35)$ had a mean age of 64 years; $57 \%$ of them were male. Almost $70 \%$ of the nonsurvivors had comorbidities, including hypertension (51.4\%), diabetes $(14.3 \%)$, or others $(34.3 \%)$. Concentrations of cardiac biomarkers in non-survivors were significantly higher than those of survivors. Compared with survivors, non-survivors had a statistically higher value of creatinine kinase-myocardial band (CK-MB; $15.64 \pm 5.50$ vs $5.39 \pm 0.75 ; p<0.001)$. The concentration

\footnotetext{
* Correspondence: 2014982567@qq.com; wuquandeng@gmail.com; 3400164739@qq.com; ypeng@anest.ufl.edu

${ }^{\dagger}$ Minghua Su and Jieru Peng contributed equally to this work.

${ }^{3}$ Department of Anesthesiology, Chengdu Women and Children's Central Hospital, School of medicine, University of Electronic Science and Technology of China, Chengdu, Sichuan, People's Republic of China ${ }^{4}$ Department of Endocrinology and Nephrology, Chongqing University Central Hospital \& Chongqing Emergency Medical Center, No.1 Jiankang Road, Yuzhong District, Chongqing 400014, People's Republic of China ${ }^{5}$ Department of Intensive Care Unit, The Fifth Hospital of Wuhan, Wuhan 610091, People's Republic of China

${ }^{6}$ Department of Anesthesiology, University of Florida College of Medicine, Gainesville, FL 32610-0254, USA

Full list of author information is available at the end of the article
}

of creatine kinase was dramatically increased in nonsurvivors (991.85 \pm 368.71 vs $48.95 \pm 5.51 ; p<0.001)$. The mean consistence of lactate dehydrogenase in nonsurvivors was three times greater than that in survivors $(653.94 \pm 65.76$ vs $281.70 \pm 22.77 ; p=0.008)$. The nonsurvivors had a statistically higher value of myoglobin $(505.70 \pm 91.52$ vs $97.13 \pm 23.87 ; p=0.001)$ and Troponin I $(10.72 \pm 9.13$ vs $0.08 \pm 0.02 ; p=0.002)$ in comparison with survivors. The serum urea nitrogen, creatinine, and high-sensitivity C-reactive protein (hs-CRP) in nonsurvivors was significantly elevated in comparison with survivors $(16.54 \pm 2.45$ vs $2.87 \pm 0.58, p<0.001 ; 184.68 \pm$ 41.27 vs $63.42 \pm 6.21, \quad p<0.001 ; \quad 217.47 \pm 46.35$ vs $27.65 \pm 21.83, \quad p=0.002$, respectively). Cardiac biomarkers in non-survivors had double-peak patterns: CK$\mathrm{MB}$ peaked on the 3rd and 10th days after admission, myoglobin on the 3rd and 8th days, and Troponin I on the 5 th and 8 th days (Fig. 1).

Cardiac biomarkers (CK-MB, myoglobin, and Troponin I) in non-survivors had a double-peak phenomenon during hospitalization, which suggested that myocardial tissue may have suffered two consecutive insults.

At the early stage (first peak wave), the white cell count and neutrophil count were almost normal, and the lymphocyte counts decreased [4]. Meanwhile, infections were milder (serum hs-CRP level was low), and serum levels of myoglobin and CK-MB were inversely correlated with hs-CRP. However, at a later stage (second peak wave), the hs-CRP level continued to increase and peaked on the 7th day, which suggested a stronger inflammatory immune response, while these individuals also exhibited multiple organ dysfunction [4]. In addition, the association between myoglobin and CK$\mathrm{MB}$ was moderately positive. Most importantly, in the latest pathological report, the degeneration and necrosis of cardiomyocytes for the non-survivors were confirmed to be caused by inflammatory destruction of cytokine 

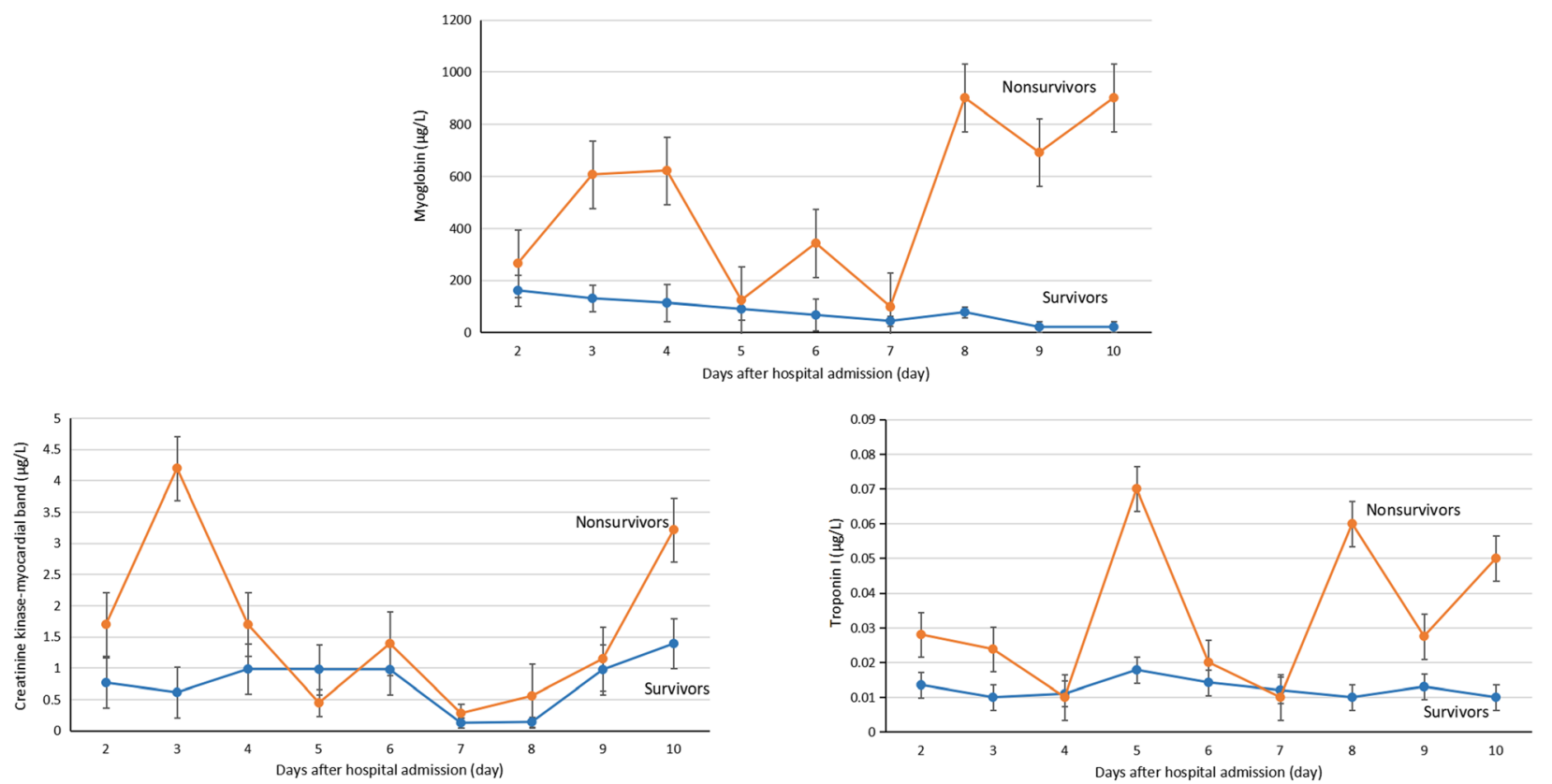

Fig. 1 Timeline chart of creatinine kinase-myocardial band (CK-MB), myoglobin, and Troponin I concentration during hospitalization comparing survivors (blue line) with non-survivors (orange line)

storm [5]. From these results, myocardial injury might be associated with the direct insult of the virus at an early stage and a subsequent attack of the cytokine cascade (sustained inflammatory response) at a later stage.

\section{Acknowledgements}

None.

\section{Authors' contributions}

The corresponding author attests that all listed authors meet the authorship criteria and that no others meeting the criteria have been omitted. MingHua Su and Jie-Ru Peng wrote the paper and conducted this study. MengJun Wu, Wu-Quan Deng, You-Sheng Yang, and Yong G. Peng designed the study and reviewed and edited the manuscript. The authors read and approved the final manuscript.

\section{Authors' information}

No

\section{Funding}

Science and Technology Department of Sichuan Province (2018SZ0224)

\section{Availability of data and materials}

All data generated or analyzed during this study are included in this published article.

\section{Ethics approval and consent to participate}

This study was only a retrospective report, so there were no patients or other participants.

\section{Consent for publication}

Not applicable.

\section{Competing interests}

The authors declare that they have no competing interests.

\section{Author details}

'Department of Emergency, Sichuan Academy of Medical Sciences \& Sichuan Provincial People's Hospital, Chengdu, Sichuan, People's Republic of China. ${ }^{2}$ Department of Medical Records Statistics, Chengdu Women and Children's Central Hospital, University of Electronic Science and Technology, Chengdu, Sichuan, People's Republic of China. ${ }^{3}$ Department of Anesthesiology, Chengdu Women and Children's Central Hospital, School of medicine, University of Electronic Science and Technology of China, Chengdu, Sichuan, People's Republic of China. ${ }^{4}$ Department of Endocrinology and Nephrology, Chongqing University Central Hospital \& Chongqing Emergency Medical Center, No.1 Jiankang Road, Yuzhong District, Chongqing 400014, People's Republic of China. ${ }^{5}$ Department of Intensive Care Unit, The Fifth Hospital of Wuhan, Wuhan 610091, People's Republic of China. ${ }^{6}$ Department of Anesthesiology, University of Florida College of Medicine, Gainesville, FL 32610-0254, USA.

Received: 16 April 2020 Accepted: 12 May 2020

Published online: 26 May 2020

\section{References}

1. Zhu N, Zhang D, Wang W, et al. A novel coronavirus from patients with pneumonia in China, 2019. N Engl J Med. 2020. https://doi.org/10.1056/ NEJMoa2001017.

2. Lancet T. Emerging understandings of 2019-nCoV. Lancet. 2020;395:311.

3. Guo T, Fan Y, Chen M, et al. Cardiovascular implications of fatal outcomes of patients with coronavirus disease 2019 (COVID-19). JAMA Cardiol. 2020. https://doi.org/10.1001/jamacardio.2020.1017.

4. Wang D, Hu B, Hu C, et al. Clinical characteristics of 138 hospitalized patients with 2019 novel coronavirus-infected pneumonia in Wuhan, China. JAMA. 2020. https://doi.org/10.1001/jama.2020.1585.

5. Zhou F, Yu T, Du R, et al. Clinical course and risk factors for mortality of adult inpatients with COVID-19 in Wuhan, China: a Retrospective Cohort Study. Lancet. 2020;395:1054-62.

\section{Publisher's Note}

Springer Nature remains neutral with regard to jurisdictional claims in published maps and institutional affiliations. 